\title{
Th. Hausen Die Akzeptanz der Peak-Flow-Messung in der Praxis
}

\author{
Acceptance of Peak-Flow Measurement in Out-Patients
}

\section{Zusammenfassung}

Die PEF-Messung bietet eine Reihe von Indikationen, wobei die wichtigste sicher die Langzeitbetreuung von Asthmatikern ist. Leider wird diese Messung nicht von allen Patienten regelmäßig durchgeführt. Eine Befragung bei Patienten, die mit einem PEFGerät ausgerüstet waren, sollte die Akzeptanz klären helfen. Methode: 65 Patienten, denen ein PEF-Gerät verordnet worden war, wurden angeschrieben und gebeten, anonym einen Fragebogen zu beantworten. Ergebnis: 49 Patienten (24 Frauen und 24 Männer, 1 ohne Angabe) beantworteten den Fragebogen. 19 Patienten messen noch, 30 Patienten haben die Messung wegen fehlender Beschwerden eingestellt. Die Messung wird regelmäßig von 14 und unregelmäßig von 4 , am häufigsten täglich $(n=9)$, seltener nur wöchentlich, monatlich oder nur gelegentlich vorgenommen. Die Messung erfolgt überwiegend morgens $(n=8), 3$ x pro Tag $(n=5)$ und $2 \times$ pro Messzeitpunkt $(n=9)$. Vor der Inhalation eines Bronchospasmoloytikums haben 11 Patienten, sowohl vor und nach der Inhalation 8 Patienten gemessen. Protokolliert wurden die Werte von 7. Als Gründe für die Messung wurden überwiegend Kontrolle und Beschwerden angegeben. Schlussfolgerung: Die PEF-Messung wird offensichtlich akzeptiert, wenn der Patient die Notwendigkeit der Messungen einsieht, z.B. für einen kurzen Zeitraum zum Zeitpunkt von Beschwerden oder Therapieein- oder -umstellungen. Unter diesen Voraussetzungen sind die meisten Patienten zu regelmäßigen, täglichen Messungen zwei bis drei Mal pro Tag und Messzeitpunkt, eventuell auch mit Protokollierung, bereit.

\section{Abstract}

There are a lot of indications for PEF-measurement of which the long-time observation of asthmatics is the most important one. Unfortunately not all patients are measuring regularly. The acceptance of PEF-measurement in patients who have got a PEFmonitor should be found out by a questioning in the following trial. Method: 65 patients who have got a prescription for a PEFmonitor since 1995 were asked to answer a questionnaire anonymously. Results: 49 patients (24 female, 24 male, 1 without information) answered the questionnaire. 19 patients are still measuring, 30 patients stopped measuring because of lacking complaints. Patients measured regularly $(\mathrm{n}=14) .4$ irregularly, most frequently daily $(\mathrm{n}=9$ ), more rarely only weekly, monthly or casually, Measuring was done mostly in the morning $(\mathrm{n}=8), 3$ times a day $(\mathrm{n}=5), 2$ times at same time $(\mathrm{n}=9) .11$ patients measured before inhalation of a beta-agonist, 8 patients before and after inhalation as well. 7 patients recorded the values. Causes for measuring mostly were control and complaints. Conclusion: PEF-Measuring obviously will be accepted if the patient understands the necessity, f.e. for a short period of time at a moment of complaints or start or change of therapy. Under those circumstances most of our patients will be ready to do PEF-measurement regularly and daily and two to three times a day and at the same time and eventually with recording the values.

\section{Einleitung}

Die Bedeutung der PEF-Messung für die Betreuung von Patienten mit variabler Atemwegsobstruktion ist weltweit unumstritten. Jeder Arzt, der diese einfache Untersuchung regelmäßig in seine
Überlegungen einbezieht, wird von deren Bedeutung zunehmend überzeugt sein und sie dementsprechend einsetzen wollen. Auch viele Patienten sehen die Möglichkeit zur Selbstbeurteilung und nutzen diese Untersuchung. Es existieren eine Reihe von Indikationen für die PEF-Messung; diese können unterschie- 
den werden nach dem Nutzen für den betreuenden Arzt in der Praxis, den Patienten und die Wissenschaft.

Die größte Bedeutung besitzt die PEF-Messung bei der Langzeitbetreuung von Patienten mit Asthma. Eines der Ziele der modernen Asthmatherapie besteht darin, die für den Patienten möglichst optimale Therapie bei gleichzeitiger Einfachheit in der Anwendung zu finden. Der behandelnde Arzt kann mit Hilfe der PEF-Messung die Therapie in der Einstellungsphase, beim eventuell möglichen Step-down oder notwendigen Step-up optimal titrieren.

Ein weiteres Ziel der modernen Asthmatherapie ist das Selbstmanagement des Patienten. Nach ausreichender Schulung soll dieser in der Lage sein, seinen aktuellen Zustand richtig einschätzen und darauf reagieren zu können. Vor allem die PEFMessung soll dem Patienten diese Selbstbeobachtung im beschwerdefreien oder zumindest -armen Intervall und ganz besonders die Beurteilung einer Selbstbehandlung anlässlich einer Verschlechterung quantitativ ermöglichen. Die Therapieempfehlungen der verschiedenen Fachgesellschaften (Tab. 1) beinhalten u. a. auch Empfehlungen zur PEF-Messung.

Optimal wären Messungen morgens, mittags und abends sowie zusätzlich zum Zeitpunkt von Beschwerden, gegebenenfalls vor und nach Inhalation eines bronchospasmolytisch wirksamen Atemwegstherapeutikum einschließlich regelmäßiger Protokollierung. Wir wissen aber, dass dieser Wunsch unrealistisch ist. Eine Patientenbefragung sollte dazu beitragen, die Akzeptanz der Messung bei Patienten zu klären.

\section{Methode}

Im Frühjahr 2001 wurden 65 Patienten, die wegen ihres Asthmas seit 1995 ein PEF-Gerät rezeptiert bekommen und in der Zeit ab 1. 1. 2000 noch Kontakt zur Praxis hatten, per Post gebeten, anonym einen Fragebogen zur PEF-Messung zu beantworten. Vertreten waren Patienten mit einem Asthma der Schweregrade 2-4, darunter zahlreiche Patienten, die durch eine erfolgreiche Dauerbehandlung in leichtere Schweregrade überführt werden konnten. Namentliche Rückmeldungen waren erbeten, aber nicht gefordert, Kommentare erwünscht.

\section{Ergebnisse}

Der Fragebogen wurde von 49 Patienten (24 Männer, 24 Frauen, $1 \times$ ohne Angabe) beantwortet (Tab. 2). Das Alter variierte von 11 bis 68 und lag im Mittel bei 43 Jahren. Die Messung wird von 19 Patienten (7 Männer, 12 Frauen) immer noch durchgeführt (im weiteren Text Gruppe A). 30 der Befragten (17 Männer, 12 Frauen, $1 \times$ ohne Angabe) haben die Messung eingestellt (im weiteren Text Gruppe B), wobei als Begründung ausschließlich fehlende Beschwerden angegeben wurden. Nur ein Patient gab zusätzlich an, vielleicht auch zu bequem zu sein. Das Durchschnittsalter lag in Gruppe A bei 45 und in Gruppe B bei 43 Jahren. Regelmäßig wird die Messung in Gruppe A von 14 und unregelmäßig von 4 durchgeführt. Frauen scheinen eher regelmäßig zu messen (Tab.3). Gemessen haben täglich 9, wöchentlich 4 (1× Doppel-
Tab. 1 Empfehlungen zur PEF-Messung der Fachgesellschaften [15]

$\begin{array}{ll}\begin{array}{l}\text { Southhampton Guidelines } 1989 \\ \text { Kanada Guidelines } 1990\end{array} & \text { Morgenwert } \\ \text { Intern. Consensusreport 1992 } & \text { Vor oder nach Beta-Stimulation } \\ \text { GINA-Richtlinien 1995/1998 } & 2 \times / \text { Tag vor und nach Beta-Stimulation } \\ \text { US-NAEPP 1997 } & \begin{array}{l}\text { Vor und nach Beta-Stimulation } \\ \text { Gibson } 1995\end{array} \\ \begin{array}{l}2 \times / \text { Tag je 3 Werte vor und nach } \\ \text { Beta-Stimulation }\end{array}\end{array}$

Tab. 2 Akzeptanz der Peak-Flow-Messung nach Verordnung eines Peak-Flow-Gerätes

\begin{tabular}{|lrrrrr|}
\hline & Männer & Frauen & keine Angabe & gesamt \\
\hline Gruppe A & misst noch & 7 & 12 & 0 & 19 \\
\hline Gruppe B & misst nicht mehr & 17 & 12 & 1 & 30 \\
& gesamt & 24 & 24 & 1 & 49 \\
\hline
\end{tabular}

Tab. 3 Messen Sie regelmäßig oder unregelmäßig? (Angaben der Gruppe B, soweit erfolgt, für den Zeitraum, zu dem noch gemessen wurde) dies gilt auch für die folgenden Tabellen)

\begin{tabular}{|llll|}
\hline & & regelmäßig & unregelmäßig \\
\hline Gruppe $A$ & gesamt & 14 & 4 \\
& Männer & 4 & 2 \\
\hline \multirow{2}{*}{ Gruppe $B$} & Frauen & 10 & 2 \\
& gesamt & $5 / 7$ & $2 / 7$ \\
& Männer & $3 / 7$ & $1 / 7$ \\
\hline & Frauen & $2 / 7$ & $1 / 7$ \\
\hline
\end{tabular}

Tab. 4 Wie oft messen Sie?

\begin{tabular}{|lllllll|}
\hline & & täglich & wöchentlich & monatlich & gelegentlich \\
\hline \multirow{2}{*}{ Gruppe A } & gesamt & 9 & 4 & 5 & 2 \\
& Männer & 4 & 0 & 3 & 0 \\
\hline \multirow{2}{*}{ Gruppe B $B$} & Frauen & 5 & 4 & 2 & 2 \\
& gesamt & $5 / 6$ & $1 / 6$ & 0 & $1 / 6$ \\
& Männer & $4 / 6$ & $1 / 6$ & 0 & 0 \\
& Frauen & $1 / 6$ & 0 & 0 & 1 \\
\hline
\end{tabular}

nennung für täglich + wöchentlich) und monatlich 5 (Tab. 4). Gelegentlich gemessen haben 2 Frauen.

Der überwiegende Teil der Befragten (Tab.5) misst morgens $(n=8)$, gefolgt von 3-mal täglicher Messung $(n=5)$, morgens und abends $(n=4)$ und abends $(n=1)$. Die Häufigkeit der Messungen pro Messzeitpunkt (Tab.6) wurde mit 2-mal $(n=9)$, gefolgt von 3-mal $(n=5)$ und nur einmaliger Messung $(n=3)$ angegeben. Mehr als 3-mal pro Messzeitpunkt hat keiner der Befrag- 
Tab. 5 Wann messen Sie?

\begin{tabular}{|c|c|c|c|c|c|c|c|}
\hline & & $a$ & $b$ & c & $d$ & $e$ & $f$ \\
\hline & & morgens & mittags & abends & $A+b$ & $A+c$ & $A+b+c$ \\
\hline \multirow{2}{*}{ Gruppe A } & Männer & 3 & 0 & 0 & 0 & 1 & 2 \\
\hline & Frauen & 5 & 0 & 1 & 0 & 3 & 3 \\
\hline Gruppe B & gesamt & $1 / 7$ & 0 & 0 & $1 / 7$ & 0 & $5 / 7$ \\
\hline
\end{tabular}

Tab. 6 Wie oft messen Sie pro Messzeitpunkt?

\begin{tabular}{llllll}
\hline & & $1 \times$ & $2 \times$ & $3 \times$ & $4 \times$ \\
\hline \multirow{2}{*}{ Gruppe A } & gesamt & $3 / 17$ & $9 / 17$ & $5 / 17$ & $0 / 17$ \\
& Männer & 1 & 3 & 3 & 0 \\
& Frauen & 2 & 6 & 2 & 0 \\
\multirow{2}{*}{ Gruppe B $B$} & gesamt & $1 / 8$ & $5 / 8$ & $2 / 8$ & 0 \\
& Männer & 0 & 4 & 1 & 0 \\
& Frauen & 1 & 1 & 1 & 0 \\
\hline
\end{tabular}

Tab. 7 Messen Sie vor und nach Inhalation?

\begin{tabular}{|llll}
\hline & & vor & vor und nach \\
\hline Gruppe $A$ & gesamt & 11 & 8 \\
& Männer & 2 & 5 \\
\hline \multirow{2}{*}{ Gruppe $B$} & Frauen & 9 & 3 \\
& gesamt & $3 / 8$ & $5 / 8$ \\
& Männer & 2 & 3 \\
\hline & Frauen & 1 & 2 \\
\hline
\end{tabular}

ten gemessen. Ausschließlich vor der Inhalation haben 11 Patienten, überwiegend Frauen, vor und nach der Inhalation 8 Patienten (Tab. 7) gemessen. Eine Protokollierung (Tab. 8) wurde nur von 7 Patienten vorgenommen, 9 hielten eine Protokollierung für überflüssig. Die meisten Befragten $(n=11)$ nutzten die Messung zur Kontrolle ihres Befindens oder bei Beschwerden oder sowohl zur Kontrolle als auch bei Beschwerden (Tab.9). Ein Mann der Gruppe A glaubte, mit Hilfe der Messung den optimalen Zeitpunkt für die Reduktion der systemischen Kortisondosis besser bestimmen zu können.

Einige Patienten der Gruppe B haben zusätzlich die Fragen für den Zeitraum beantwortet, zu dem sie noch gemessen haben. Die Patienten haben überwiegend regelmäßig (5/6) dreimal pro Tag (5/7) oder morgens oder abends (je $1 \times$ ) zweimal pro Messzeitpunkt (5/8) vor und nach Inhalation (5/8) gemessen und diese Werte auch protokolliert (5/7). Die Motivation für die Messung entsprach derjenigen in Gruppe A.
Tab. 8 Protokollieren Sie die Werte?

\begin{tabular}{llll}
\hline & & ja & nein \\
\hline Gruppe $A$ & gesamt & $7 / 17$ & $10 / 17$ \\
& Männer & 4 & 3 \\
\hline \multirow{2}{*}{ Gruppe B } & Frauen & 3 & 7 \\
& gesamt & $5 / 7$ & $2 / 7$ \\
& Männer & 4 & 0 \\
\hline & Frauen & 1 & 2 \\
\hline
\end{tabular}

Tab. 9 Zu welcher Gelegenheit messen Sie?

\begin{tabular}{|llllll}
\hline & & Kontrolle & $\begin{array}{l}\text { bei Beschwer- } \\
\text { den }\end{array}$ & $\begin{array}{l}\text { Kontrolle + } \\
\text { Beschwerden }\end{array}$ & ? \\
\hline Gruppe A & gesamt & $11 / 18$ & $1 / 18$ & $5 / 18$ & $1 / 18$ \\
& Männer & 4 & 1 & 0 & 1 \\
\hline & Frauen & 7 & 0 & 5 & 0 \\
\hline Gruppe B & gesamt & $4 / 9$ & $5 / 9$ & $0 / 9$ & $0 / 9$ \\
& Männer & 4 & 2 & 0 & 0 \\
\hline & Frauen & 0 & 3 & 0 & 0 \\
\hline
\end{tabular}

Diskussion

Autoritäre Anordnungen gehören der Vergangenheit an. Eine möglichst große Compliance ist nur zu erreichen, wenn der Patient die Notwendigkeit zur Therapie einsieht, d. h. seine Krankheit anerkennt und somit eine Therapiebereitschaft geweckt werden kann. Schulungen und die Möglichkeit zur Selbstbehandlung im angemessenen Rahmen machen unsere Patienten mit ihrer Krankheit vertraut. Dem Ziel einer guten Patienten-Compliance sind wir durch die Schulung und einfache Therapieregime mit niedriger Anwendungsfrequenz dank moderner Substanzen und Darreichungsformen immer näher gekommen. Auf diese Weise sind die Beschwerden der Asthmatiker immer geringer geworden, so dass zahlreiche Patienten dazu neigen, ihre erfolgreiche Therapie eigenmächtig abzubrechen oder zu reduzieren.

Es ist nicht zeitgemäß, wenn wir von unseren Patienten dauerhaft ein- bis mehrfache PEF-Messungen pro Tag mit regelmäßiger Protokollierung erwarten. Auf der einen Seite sehen wir un- 
sere Patienten als Partner, denen wir eine Selbstbehandlung erlauben, auf der anderen Seite machen wir uns aber als Ärzte unglaubwürdig, wenn wir eine Eigenverantwortlichkeit der Patienten bei der Notwendigkeit und Durchführung der PEF-Messung ablehnen.

Die Ergebnisse der vorliegenden Untersuchung geben die Vorstellungen unserer Patienten zur PEF-Messung wieder $[6,10]$. Es muss an dieser Stelle auch erwähnt werden, dass in der Praxis des Autors allen Atemwegskranken eine Schulung [7-9] mit einer Akzeptanz von ca. 45\% [8] angeboten wird, in deren Verlauf die Patienten u. a. auf die Bedeutung der PEF-Messung hingewiesen werden, PEF-Kurven zu interpretieren lernen und Exazerbationen eines Asthmas zu den ganz seltenen Ereignissen zählen. Die Ergebnisse der Befragung erfahren eine gewisse Einschränkung, weil die anonyme Befragung leider keine Aussage über die Dauer der Messungen und eine Überprüfung der Angaben anhand von PEF-Protokollen ermöglichte.

Der überwiegende Teil der Patienten $(30 / 49=61 \%)$ hat die PEFMessung eingestellt. In einer ähnlichen Befragung [20] bestätigen 52 von 120 Befragten $(=43 \%$ ) die Einstellung der Messung. Männer beenden die PEF-Messung schneller, Frauen setzen die Messungen länger fort. Die Kommentare der aktuell Befragten belegen, dass diese mit Erreichen eines Therapieoptimums und stabilen PEF-Werten den Sinn der Messung nicht mehr eingesehen haben. Diese Feststellung belegt gleichzeitig, dass die in den Therapieempfehlungen gesteckten Ziele, Beschwerdefreiheit unter regelmäßiger Dauertherapie, erreicht werden können. In beiden Untersuchungen haben einige der Befragten die Befragung als erneute Motivation zur Messung angesehen. Einige der aktuell Befragten haben nach mündlichen Hinweisen die Messung bei stabilen Werten aber wieder eingestellt.

Starre Messvorgaben lassen sich allenfalls für einen begrenzten Zeitraum aufrechterhalten. In der Praxis sind dies die Phasen der Therapieeinstellung oder -änderungen. Cote [2] berichtet von einer Compliance von $63 \%$ bei Messungen über 1 Monat, die dann in 6 Monaten auf 50\% und 12 Monaten auf 33\% sank. Die Bereitschaft zur Messung ist offensichtlich höher, wenn die Daten regelmäßig überprüft und die Kenntnisse aufgefrischt werden [16]. Unter den Bedingungen optimaler Betreuung (angemessene medizinische Versorgung, individuelle Schulung in Selbstbehandlung und regelmäßige Vorstellungen) belegt die PEF-Messung nur bei Patienten mit mittelgradigem Asthma einen positiven Effekt auf vorgegebene Empfehlungen [13]. Die meisten Patienten scheinen ihre Therapieentscheidungen allerdings auch nach eingehender Schulung lieber von ihren Beschwerden als von PEF-Messungen abhängig zu machen [3].

Abgesehen von der fehlenden Bereitschaft zur regelmäßigen Messung ist die Glaubwürdigkeit der Messungen bei Kindern bereits bei nur 3- bis 5 -wöchiger Messung anzuzweifeln $[1,11,18]$. Messungen über einen Zeitraum von nur 2 Wochen haben bei Erwachsenen noch akzeptable Daten geliefert [4].

Die Patienten dieser Befragung, die die PEF-Messung aus Überzeugung oder wegen des Krankheitsverlaufes aus Notwendigkeit weiter durchgeführt haben, haben die Messung entsprechend unseren Vorstellungen durchgeführt. Überwiegend erfolgte die Messung regelmäßig, morgens oder morgens und abends, somit zu akzeptablen Tageszeiten, 2-3-mal pro Messzeitpunkt und eventuell auch vor und nach Inhalation einer bronchospasmolytisch wirksamen Substanz. Eine Protokollierung erfolgte seltener. Für die zweimalige Messung fanden Hamid et al. [4] eine Compliance von $67 \%$ für morgens und $72 \%$ für abends bei einer Studiendauer von nur 2 Wochen. Bei einer Studiendauer von 5 Wochen sank die Messfrequenz in der 5. Woche auf 1-mal pro Tag bei Kindern [1]. Leider lässt die vorliegende anonyme Befragung keine Aussage über die Dauer der Messungen zu.

Die Messungen erfolgten fast ausnahmslos zur Kontrolle oder bei Beschwerden. In einem Fall wurde versucht, mit Hilfe der PEF-Messung den Zeitpunkt für eine Reduktion der Kortisondosis besser bestimmen zu können.

Bei der Behandlung von Asthmatikern haben wir die Compliance durch effektivere und einfachere Therapieregime verbessern können. Eine größere Bereitschaft zur PEF-Messung kann nur erreicht werden, wenn Empfehlungen zur PEF-Messung herausgegeben werden, die vom behandelnden Arzt und Patienten verstanden und als sinnvoll anerkannt werden, und die im täglichen Leben praktikabel sind. Dazu zählen die zeitliche Limitierung $[2,14]$ und eine gute medizinische Versorgung einschließlich Schulung mit Empfehlungen zur Selbstbehandlung [3,12].

\section{Schlussfolgerung}

Nach den Ergebnissen der Befragung sowie der wenigen vorhandenen Studien in der Literatur sind Empfehlungen zur PEF-Messung akzeptabel, wenn die Messungen

- zeitlich begrenzt, d.h. zum Zeitpunkt einer Therapieeinstellung, -umstellung oder-optimierung,

- ein- bis 2-mal pro Tag, d.h. morgens und abends,

- möglichst 2-3-mal pro Messzeitpunkt,

- eventuell bei Verwendung eines bronchospasmolytisch wirkenden Therapeutikums vor und nach Inhalation,

- mit Protokollierung erfolgen sollen.

Zusätzliche Messungen sollten im beschwerdefreien Intervall zur Überprüfung des subjektiven Empfindens empfohlen werden.

\section{Literatur}

${ }^{1}$ Burkhart PV, Dunbar-Jacob JM, Rohey JM. Accuracy of children' self reported adherence to treatment. J Nurs Scholarsh 2001; 33: 27-32

2 Cote J, Cartier A, Malo JL, Rouleau M, Boulet L-P. Compliance with peak expliratory monitoring in home management of asthma. Chest 1998; 113/4: 968 - 972

${ }^{3}$ Garrett J, Fenwick JM, Taylor G, Mitchell E, Rea H. Peak expiratory flow meters (PEFMs) - who uses them and how does education affect the pattern of utilisation? Australian and New Zealand Journal of Medicine 1994; 24: $521-529$

${ }^{4}$ Hamid S, Corden ZM, Ryan DP, Burnett I, Cochrane GM. Evaluation of an electronic hand-help spirometer in patients with asthma. Respir Med. 1998; 92: 1177-1180

${ }^{5}$ Hausen Th. Atemwegserkrankungen, Asthma, Chronische Bronchitis, Emphysem. Aus der Praxis für die Praxis. Berlin: Ullstein Mosby $\mathrm{GmbH}, 1993$

${ }^{6}$ Hausen Th. Praktische Pneumologie (Pneumologische Kasuistiken unter besonderer Berücksichtigung von Lungenfunktion und Peak-FlowMessung). Kirchheim Verlag, 1996 
${ }^{7}$ Hausen Th. Schulung von Atemwegskranken in der Praxis Evaluation und Information über Probleme bei der Evaluation. Präv-Rehab 1996; 8: $47-52$

${ }^{8}$ Hausen Th. Ambulante Kurzschulung im Vergleich zu Kombination aus Kurz- und Gruppenschulung bei Patienten mit chronisch-obstruktiver Atemwegserkrankung. Präv-Rehab 1998; 10: 83-88

${ }^{9}$ Hausen Th. Patientenschulung - woran ist ein Langzeiteffekt abzulesen und wie lange hält dieser an? Pneumologie 1999; 53: 289-295

${ }^{10}$ Hausen Th. Zwei nicht alltägliche (?) Kasuistiken einer Asthmaerkrankung. Pneumologie 2000; 54: 116-122

${ }^{11}$ Kamps AW, Roorda RJ, Brand PL. Peak flow diaries in childhood asthma are unrealiable. Thorax 2001; 56: 180-182

12 Lopez-Vina A, Del Castillo-Arevalo F. Influence of peak flow monitoring on an asthma self-management education programme. Respir Med 2000; 94: 760-766

${ }^{13}$ Malo JL. Assessment of peak flow exspiratory flow in asthma. Current Opinion in Pulmonary Medicine 1996; 2: 75-80

${ }^{14}$ Malo JL, Trudeau C, Ghezzo H, L’Archeveque J, Cartier A. Do subjects investigated for occupational asthma through serial peak exspiratory flow measurements falsify their results? Revue Francaise d'Allergologie et d'Immunologie Clinique 1996; 36: 393 - 399
${ }^{15}$ Mendenhall AB, Tsien AY. Evaluation of physician and patient compliance with the use of peak flow meters in commercial insurance and Oregon health plan asthmatic populations. Ann Allergy, Asthma Immunol 2000; 84: 523 - 527

${ }^{16}$ Murata GH, Kapsner CO, Lium DJ, Busby HK. Patient compliance with peak flow monitoring in chronic obstructive pulmonary disease. Am J Med Sci 1998; 315: 296-301

${ }^{17}$ Rabe KF, Vermeire PA, Soriano JB, Maier WC. Clinical managment of asthma in 1999: the Asthma Insights and Reality in Europe (AIRE) study. Eur Respir J 2000; 16: $802-807$

18 Redline S, Wright EC, Kattan M, Kercsmar C, Weiss K. Short-term compliance with peak flow monitoring: results from a study of inner city children with asthma. Ped Pulmon 1996; 21: $203-210$

${ }^{19}$ Rothe T. Asthma-Selbst-Management mit dem Ampelschema: Welcher Peak-Flow, welche Guidelines? Pneumologie 1999; 53: 626 - 629

${ }^{20}$ Ueshima Y, Kikuchi T, Kaneko N, Kaneshige H, Suzuki H. Study of how to continue peak flow monitoring for patients with bronchial asthma: questionnaire to continuation and interruption patients Aerugi-Japanese. Journal of Allergology 2000; 49: 420-427 